\title{
Determination of Transformer Oil Quality Parameters by its Color Coordinates
}

\author{
Kozlov V.K., Valiullina D.M., Kurakina O.E., Sadykov E.M. \\ Kazan State Power Engineering University, \\ Kazan, Russian Federation
}

\begin{abstract}
The service life of transformers and, as a consequence, of the electric power systems depends largely on the chemical composition and electrical insulating properties of the oil. In this regard, much attention is paid to the control of physical and chemical indicators and operational properties of transformer oils. The aim of this work is to determine the important diagnostic parameters such as the acid number and the tangent of the dielectric loss angle of the transformer oil based on the measured coordinates of the color of the transformer oil. This goal is achieved by the study of the coordinates of the transformer oils chromaticity. For this purpose, a white light source is used, which is a LED with a pumping line of $450 \mathrm{~nm}$ and a maximum radiation in the region of $550-560 \mathrm{~nm}$, with radiation in the range from 400 to $800 \mathrm{~nm}$. Sensors 1, 2, 3 allow determining the chromaticity coordinates of the measured source R, G, B (red, green, blue), due to several receivers having spectral characteristics of sensitivity in the corresponding region of the visible spectrum. The most significant result of the work was the establishment of correlations between the TM chromaticity coordinates 6 their acid number and the tangent of the dielectric loss angle of TM. The significance of the results obtained was that the acid number and the tangent of the dielectric loss angle of the transformer oil could be determined by the chromaticity coordinates, and hence, by the color of the oil.
\end{abstract}

Keywords: transformer oil, acid number, dielectric loss tangent, color characteristics, chromaticity coordinates

DOI: https://doi.org/10.52254/1857-0070.2021.3-51.03

UDC: 621.315.615.22

\section{Determinarea parametrilor de calitate ai uleiului de transformator prin coordonate de cromaticitate}

Kozlov V.K., Valiullina D.M., Kurakina O.E., Sadâkov Ă.M.

Universitatea de Stat de energetică din Kazani,

Kazani, Federația Rusă

Rezumat. Durata de viață a transformatoarelor și, ca o consecință a sistemului electroenergetic, depinde în mare măsură de compoziţia chimică și de proprietățile izolatoare electrice ale uleiului. În acest sens, se acordă o atenție deosebită controlului indicatorilor fizico-chimici și proprietăţilor de performanţă ale uleiurilor de transformare. Scopul lucrării este de a determina parametri de diagnostică atât de importanți precum numărul acidului și tangenta unghiului de pierdere dielectric al uleiului de transformator pe baza coordonatelor de cromaticitate măsurate ale uleiului de transformator, adică după culoarea uleiului. Acest obiectiv este atins prin studierea coordonatelor cromaticității uleiurilor de transformare. Pentru aceasta, se utilizează o sursă de lumină albă, care este un LED cu o linie de pompare de $450 \mathrm{~nm}$ și o radiație maximă în regiunea de 550-560 nm, cu radiație în regiune de la $400 \mathrm{~nm}$ la $800 \mathrm{~nm}$. Senzorii 1, 2, 3 permit determinarea coordonatelor de cromaticitate ale sursei măsurate $\mathrm{R}, \mathrm{G}, \mathrm{B}$ (roșu, verde, albastru) datorită mai multor receptoare cu caracteristici spectrale de sensibilitate în regiunea corespunzătoare a spectrului vizibil. Această instalație vă permite determinarea coordonatelor cromaticității radiației de luminiscență TM (senzor 1), radiaţia împrăștiată (senzor 2) şi radiaţia transmisă (senzor 3) și astfel obținerea caracteristicilor cantitative ale acestor emisii. Cel mai semnificativ rezultat al articolului este stabilirea corelaţiilor dintre coordonatele de cromaticitate ale TM, numărul lor de acid şi tangenta unghiului de pierdere dielectric al TM. Semnificaţia rezultatelor obținute constă în faptul că numărul acidului şi tangenta unghiului de pierdere dielectric al uleiului de transformator pot fi determinate de coordonatele de cromaticitate și, în consecință, de culoarea uleiului.

Cuvinte-cheie: ulei de transformator, numărul acidului, tangenta pierderii dielectrice, caracteristicile culorii, coordonatele cromatice.

(C) Kozlov V.K., Valiullina D.M., Kurakina O.E., Sadykov E.M. 2021 


\section{Определение параметров качества трансформаторного масла по его координатам цветности}

Козлов В.К., Валиуллина Д.М., Куракина О.Е., Садыков Э.М. ФГБОУ ВО Казанский государственный энергетический университет, Казань, Российская федерация

Аннотащия. Силовые маслонаполненные трансформаторы являются неотъемлемой частью электроэнергетической системы. Длительность срока службы трансформаторов и как следствие электроэнергетической системы во многом зависит от химического состава и электроизоляционных свойств масла. В связи с этим большое внимание уделяется контролю физико-химических показателей и эксплуатационных свойств трансформаторных масел. Целью работы является определение таких важных диагностических параметров, как кислотное число и тангенс угла диэлектрических потерь трансформаторного масла по измеренным координатам цветности трансформаторного масла, т.е. по цвету масла. Поставленная цель достигается за счет изучения координат цветности трансформаторных масел. Для этого используется источник белого света, в качестве которого выступает светодиод с линией накачки 450 нм и максимумом излучения в районе 550-560 нм, с излучением в области от 400 нм до 800 нм. Датчики 1, 2, 3 позволяют определять координаты цветности измеряемого источника R, G, B (красный, зеленый, синий) за счет нескольких приемников имеющих спектральные характеристики чувствительности в соответствующей области видимого спектра. Данная установка позволяет определять координаты цветности излучения люминесценции ТМ (Датчик 1), рассеянное излучение (Датчик 2) и прошедшее излучение (Датчик 3) и тем самым получать количественные характеристики этих излучений. Наиболее существенным результатом статьи является установление корреляционных связей между координатами цветности ТМ их кислотным числом и тангенсом угла диэлектрических потерь ТМ. Значимость полученных результатов состоит в том, что по координатам цветности, а, следовательно, и по цвету масла можно определить кислотное число и тангенс угла диэлектрических потерь трансформаторного масла. Это значительно облегчает диагностику трансформаторного масла, дает ценную информацию относительно его текущего состояния, позволяет предотвратить его полное окисление и как следствие возможные причины аварий маслонаполненного оборудования.

Ключевые слова: трансформаторное масло, кислотное число, тангенс угла диэлектрических потерь, цветовые характеристики, координаты цветности.

\section{ВВЕДЕНИЕ}

Трансформаторное масло (ТМ) является основным элементом стабильной работы высоковольтных силовых трансформаторов, да и электроэнергетических систем в целом. Старение ТМ оказывает значительное влияние на сроки и условия эксплуатации силового маслонаполненного оборудования [1-4]. Состояние масла оценивается по результатам испытаний проб масла, отобранных с учетом режима работы и температуры трансформатора из нескольких точек. По экспертным оценкам анализ масла позволяет выявить около $70 \%$ возможных дефектов в трансформаторах [3,5,6]. Ocновными характеристиками трансформаторных масел являются: пробивное напряжение (зависит от загрязнения масла водой, воздухом, или другими примесями), кислотное число, тангенса угла диэлектрических потерь, содержание воды в масле; температура вспышки, температура застывания, температура замерзания, кинематическая вязкость, внешний вид (цвет, прозрачность, отсутствие примесей) [7-10].

В нашем исследовании более подробно рассмотрены кислотное число (КЧ) и тангенс угла диэлектрических потерь при $90^{\circ}$ $\mathrm{C}, \%\left(\operatorname{tg} \delta 90^{\circ} \mathrm{C}, \%\right)$, являющиеся основными параметрами, характеризующими степень старения ТМ [4, 11, 12]. Вопросам изучения данных параметров посвящено множество трудов в мировой практике [1316].

Например, в статье [17] представлена модель на основе нечеткой логики для оценки остаточного ресурса силового трансформатора на основе значений поверхностного натяжения и кислотного числа изоляционного масла силового трансформатора. В работе [18] метод температурного титрования был использован для определения кислотного числа трансформаторного масла. Тангенс угла диэлектрических потерь зависит от изолирующей способности и степени старения трансформаторного масла, что является важным параметром для контроля безопасной работы трансформатора [19]. Тангенс угла диэлектрических потерь один из диагностических параметров, связанных с электростатическим зарядом, который в свою очередь вли- 
яет на характеристику пробоя ТМ [20]. В работах $[21,22]$ исследованы цветовые характеристики ТМ визуальным способом и их связь с основными параметрами, характеризующими техническое состояние ТМ, определяемыми согласно РД [16].

\section{МЕТОДЫ, РЕЗУЛЬТАТЫ И ОБСУЖДЕНИЕ}

Для изучения были взяты образцы ТМ, отобранных из работающих силовых трансформаторов в АО «Сетевая компания» РТ, характеристики которых определялись в их аттестованных лабораториях.

Таблица 1.

(Table 1)

Характеристики трансформаторных масел.

(Characteristics of transformer oils)

\begin{tabular}{|c|c|c|c|c|c|c|c|}
\hline $\begin{array}{c}\text { № пробы } \\
\text { (Sample no) }\end{array}$ & $\begin{array}{c}\text { Кислотное } \\
\text { число } \\
\text { (Acid } \\
\text { number) } \\
\end{array}$ & $\begin{array}{c}\operatorname{tg} \delta 90^{\circ} \mathrm{C} \\
\% \\
\left(\operatorname{tg} \delta 90^{\circ}\right. \\
\mathrm{C}, \%)\end{array}$ & $\begin{array}{c}\text { Класс } \\
\text { чистоты } \\
\text { (Cleanliness } \\
\text { class) } \\
\end{array}$ & $\begin{array}{l}\mathrm{U}_{\text {np }} \\
\left(\mathrm{U}_{\mathrm{bt})}\right)\end{array}$ & $\begin{array}{c}\text { ВРК и Щ } \\
\text { (WSA and A) }\end{array}$ & $\begin{array}{c}\mathrm{H}_{2} \mathrm{O} \\
\left(\mathrm{H}_{2} \mathrm{O}\right)\end{array}$ & $\begin{array}{l}\mathrm{t}^{\mathrm{o}}{ }_{\text {всп }} \\
\left(\mathrm{t}^{\mathrm{o}} \mathrm{fp}\right)\end{array}$ \\
\hline 76 & 0,14 & 47,052 & 9 & 67,6 & 3,75 & 39,46 & 140,1 \\
\hline 74 & 0,113 & 37,751 & 10 & 65,5 & 3,9 & 46,56 & 138 \\
\hline 72 & 0,108 & 29,597 & 10 & 69,1 & 4,03 & 66,72 & 140 \\
\hline 2 & 0,1778 & 25,966 & 10 & 79,4 & & & \\
\hline 4 & 0,1037 & 9,816 & 9 & 90 & & & \\
\hline 71 & 0,084 & 10,014 & 15 & 41 & 5,4 & 46,15 & 137,9 \\
\hline 5 & 0,0372 & 3,785 & 9 & 56,7 & & & \\
\hline $\mathrm{X} 1076$ & 0,0563 & 0,904 & 7 & 83,2 & 5,85 & 16,05 & 145,9 \\
\hline 0 & 0,049 & 6,731 & 10 & 84 & & 15,29 & 142 \\
\hline 3 & 0,0539 & 5,313 & 10 & 29,6 & & & \\
\hline 73 & 0,032 & 7,756 & 10 & 43,8 & 5,85 & 38,93 & 149,9 \\
\hline 1 & 0,0217 & 1,816 & 10 & 67,1 & & & \\
\hline 27 & 0,013 & 0,557 & 7 & 86 & 5,98 & 1,67 & 151,9 \\
\hline X1074 & 0,013 & 2,107 & 9 & 79,3 & & 11,05 & \\
\hline 42 & 0,014 & 1,706 & 7 & 89,4 & 5,95 & 18,57 & 153,9 \\
\hline $\begin{array}{c}\text { X1040 (Po3 } \\
\text { №1) }\end{array}$ & 0,027 & 3,129 & 11 & 63,1 & 6,15 & 19,84 & 160,2 \\
\hline Буй $2 \mathrm{~T}$ & 0,025 & 6,962 & 10 & 77,3 & 6,15 & 13,14 & 137,9 \\
\hline $\begin{array}{l}\text { X1040(2) } \\
\text { (Cep №2) }\end{array}$ & 0,0051 & 0,526 & 7 & 70 & 6,23 & 5,95 & 147,9 \\
\hline Буй $1 \mathrm{~T}$ & 0,019 & 4,2 & 10 & 88,4 & 6,07 & 11,2 & 148 \\
\hline 61 & 0,013 & 0,255 & 10 & 34,3 & & 21,74 & \\
\hline 10 & 0,0042 & & 8 & 55,5 & 6,1 & 32,59 & \\
\hline 12 & 0 & 0,028 & 9 & 85,9 & & 10,31 & \\
\hline 75 & 0,015 & 0,456 & 12 & 28,9 & 6,01 & 30,38 & 137,9 \\
\hline $\begin{array}{c}\text { X1049-1 } \\
(11)\end{array}$ & 0 & 0,249 & 8 & 90 & 6,38 & 10,65 & 144 \\
\hline Нитро 10 & 0 & 0,145 & & 89,7 & 6,95 & 12,5 & 144 \\
\hline $\mathrm{X} 1072$ & 0 & 0,063 & 8 & 78,8 & & 5,05 & \\
\hline
\end{tabular}


В таблице 1 приведены следующие характеристики: кислотное число, тангенс угла диэлектрических потерь при $90^{\circ} \mathrm{C}$, класс чистоты (кл. чист.), пробивное напряжение $\left(U_{n p}\right)$, водорастворимые кислоты и щелочи (ВРК и Щ.), влагосодержание в г/т $\left(\mathrm{H}_{2} \mathrm{O}\right)$ и температура вспышки в закрытом тигле

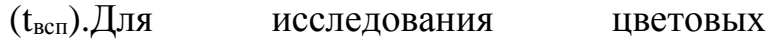
характеристик масел была собрана установка, блок-схема которой приведена на рис. 1.

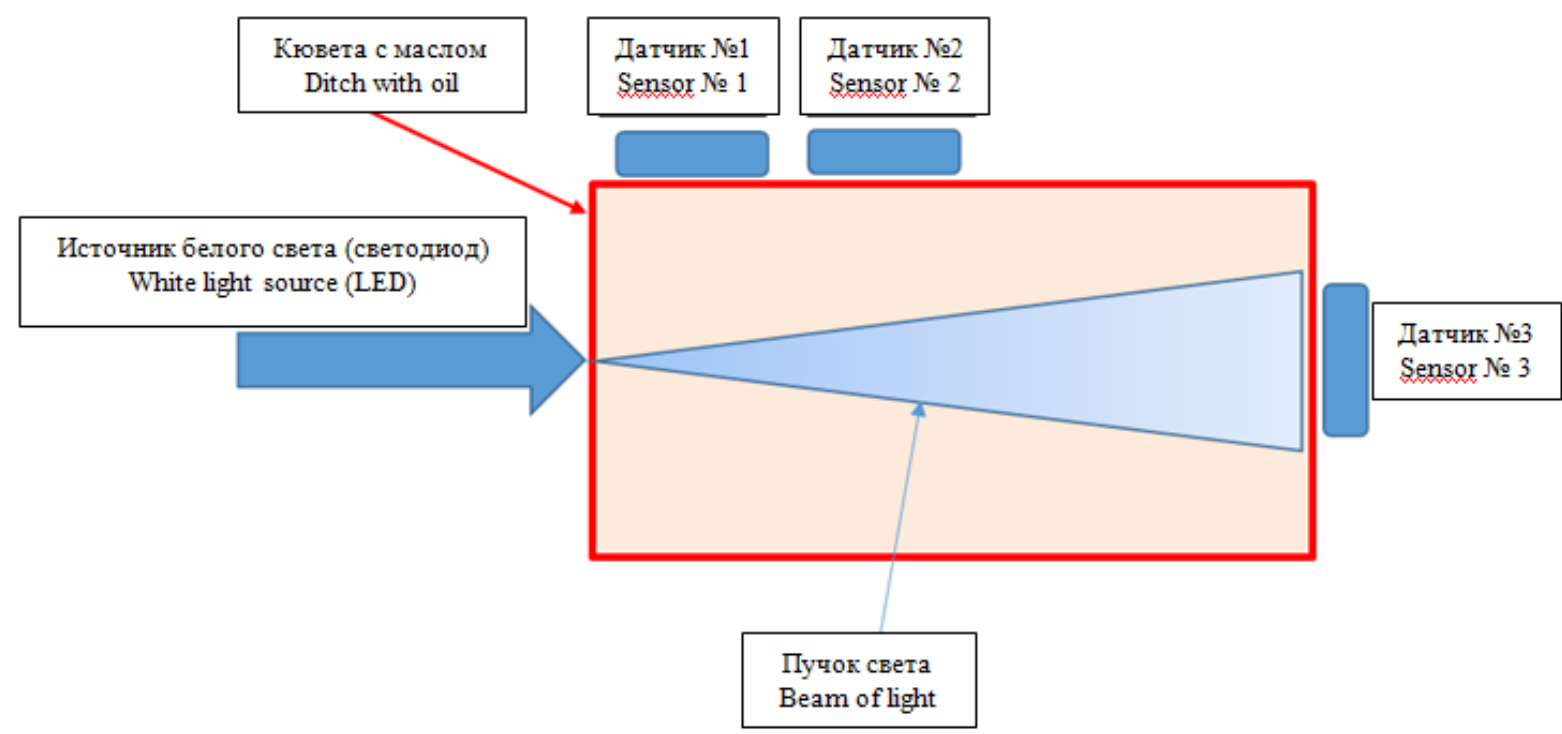

Рис. 1. Блок-схема установки для определения координат цветности ТМ.

Fig. 1. Block diagram of the installation for determining the coordinates of the TM chromaticity.

В качестве источника белого света применяется светодиод с линией накачки 450 нм и максимумом излучения в районе 550 560 нм, с излучением в области от 400 нм до 800 нм. Датчики 1, 2, 3 созданы на основе приемников TCS 3472, позволяющих определять координаты цветности измеряемого источника R, G, B (красный, зеленый, синий) за счет нескольких приемников имеющих спектральные характеристики чувствительности в соответствующей области видимого спектра. Данная установка позволяет определять согласно [23] координаты цветности излучения люминесценции ТМ (Датчик 1), рассеянное излучение (Датчик 2) и прошедшее излучение (Датчик 3) и тем самым получать количественные характеристики этих излучений в отличии от качественных приведенных в работах [21,22].

В таблице 2 приведены координаты цветности всех образцов ТМ полученных с помощью созданной установки, R1, G1, B1 -с датчиком 1, R2, G2, B2 -с датчиком 2 и R3, G3, B3 -с датчиком 3.

По полученным данным прослеживается плавное смещение цвета масла в 1, 2 и 3 области при возрастании как его кислотного числа, так и при возрастании тангенса угла диэлектрических потерь. Не имеется такой четкой зависимости при возрастании tвсп и концентрации воды в масле и совсем отсутствует такая зависимость при возрастании $U_{n p}$.

В области 1 при повышении кислотного числа цвет плавно меняется от синезеленого через зеленый, далее желтый, коричневый и красный, т.е. в сторону возрастания длины волны. Аналогичное изменение происходит с цветом масла и при возрастании $\operatorname{tg} \delta$, в указанных в табл. 1 пределах изменения $\operatorname{tg} \delta$ ТМ.

В области 2 цвет масла как при повышении кислотного числа, так и при повышении $\operatorname{tg} \delta$ повторяет изменение цвета в области 1, с учетом некоторой корректировки его за счет повышения молекулярного поглощения в начале кюветы и исключения из белого цвета источника фиолетовой и зеленой области спектра. 
Таблица 2.

(Table 2)

Координаты цветности образцов ТМ.

(Chromaticity coordinates of TM samples)

\begin{tabular}{|c|c|c|c|c|c|c|c|c|c|}
\hline ح辠 & 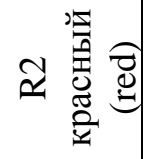 & 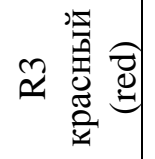 & ত & Oे & 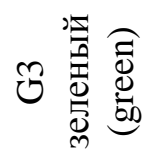 & 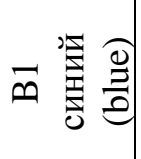 & ๗ & ๗ & 可焉 \\
\hline 0,55 & 1,00 & 0,80 & 0,27 & 0,00 & 0,15 & 0,18 & 0,00 & 0,10 & 0,049 \\
\hline 0,42 & 0,60 & 0,69 & 0,37 & 0,20 & 0,22 & 0,16 & 0,00 & 0,11 & 0,022 \\
\hline 0,53 & 1,00 & 0,89 & 0,29 & 0,00 & 0,09 & 0,18 & 0,00 & 0,13 & 0,178 \\
\hline 0,43 & 0,75 & 0,77 & 0,38 & 0,25 & 0,18 & 0,19 & 0,00 & 0,11 & 0,054 \\
\hline 0,54 & 1,00 & 0,84 & 0,31 & 0,00 & 0,11 & 0,15 & 0,00 & 0,10 & 0,104 \\
\hline 0,50 & 1,00 & 1,06 & 0,29 & 0,00 & 0,14 & 0,14 & 0,00 & 0,16 & 0,037 \\
\hline 0,19 & 0,29 & 0,51 & 0,37 & 0,41 & 0,38 & 0,39 & 0,24 & 0,17 & 0,004 \\
\hline 0,09 & 0,10 & 0,39 & 0,35 & 0,38 & 0,38 & 0,52 & 0,46 & 0,23 & 0,000 \\
\hline 0,37 & 0,60 & 0,69 & 0,37 & 0,20 & 0,22 & 0,22 & 0,20 & 0,11 & 0,013 \\
\hline 0,39 & 0,60 & 0,72 & 0,36 & 0,20 & 0,22 & 0,21 & 0,20 & 0,11 & 0,014 \\
\hline 0,28 & 0,36 & 0,52 & 0,38 & 0,36 & 0,34 & 0,30 & 0,21 & 0,15 & 0,013 \\
\hline 0,50 & 0,67 & 0,88 & 0,29 & 0,00 & 0,11 & 0,21 & 0,00 & 0,12 & 0,084 \\
\hline 0,58 & 1,00 & 0,92 & 0,33 & 0,00 & 0,09 & 0,17 & 0,00 & 0,13 & 0,108 \\
\hline 0,36 & 0,67 & 0,71 & 0,36 & 0,17 & 0,22 & 0,21 & 0,17 & 0,11 & 0,032 \\
\hline 0,55 & 1,00 & 0,93 & 0,27 & 0,00 & 0,08 & 0,18 & 0,00 & 0,14 & 0,113 \\
\hline 0,14 & 0,15 & 0,40 & 0,38 & 0,39 & 0,36 & 0,45 & 0,40 & 0,24 & 0,015 \\
\hline 0,55 & 1,00 & 0,95 & 0,27 & 0,00 & 0,09 & 0,18 & 0,00 & 0,15 & 0,140 \\
\hline 0,32 & 0,45 & 0,55 & 0,39 & 0,36 & 0,33 & 0,27 & 0,09 & 0,13 & 0,019 \\
\hline 0,33 & 0,50 & 0,58 & 0,39 & 0,30 & 0,30 & 0,25 & 0,10 & 0,12 & 0,025 \\
\hline 0,38 & 0,57 & 0,64 & 0,38 & 0,29 & 0,26 & 0,21 & 0,14 & 0,11 & 0,027 \\
\hline 0,24 & 0,50 & 0,54 & 0,41 & 0,40 & 0,33 & 0,31 & 0,10 & 0,12 & 0,005 \\
\hline 0,16 & 0,11 & 0,35 & 0,36 & 0,35 & 0,39 & 0,42 & 0,48 & 0,45 & 0,000 \\
\hline 0,12 & 0,08 & 0,34 & 0,35 & 0,35 & 0,37 & 0,49 & 0,53 & 0,33 & 0,000 \\
\hline 0,41 & 0,67 & 0,65 & 0,37 & 0,33 & 0,25 & 0,22 & 0,17 & 0,11 & 0,013 \\
\hline 0,50 & 1,00 & 0,84 & 0,25 & 0,00 & 0,09 & 0,13 & 0,00 & 0,11 & 0,056 \\
\hline 0,30 & 0,47 & 0,55 & 0,38 & 0,35 & 0,33 & 0,27 & 0,12 & 0,12 & 0,005 \\
\hline 0,37 & 0,62 & 0,74 & 0,35 & 0,23 & 0,19 & 0,24 & 0,08 & 0,11 & 0,030 \\
\hline
\end{tabular}

Полученная зависимость изменения цвета масла при возрастании кислотного числа и $\operatorname{tg} \delta$ полностью подтверждает наличие корреляционной связи между кислотным числом и тангенсом угла диэлектрических потерь ТМ установленного в [24], что объясняет природу этой корреляции аналогичным изменением молекулярного состава масла и возникновением коллоидных частиц и отсутствие подобных корреляций или их слабую связь с $U_{n p}, t_{b c n}$ и $\mathrm{H}_{2} \mathrm{O}$, как показано в [24].

На рис. 2 приведены зависимости коэффициентов R, G, В от кислотного числа 
TM, которые имеют сильную линейную корреляцию.
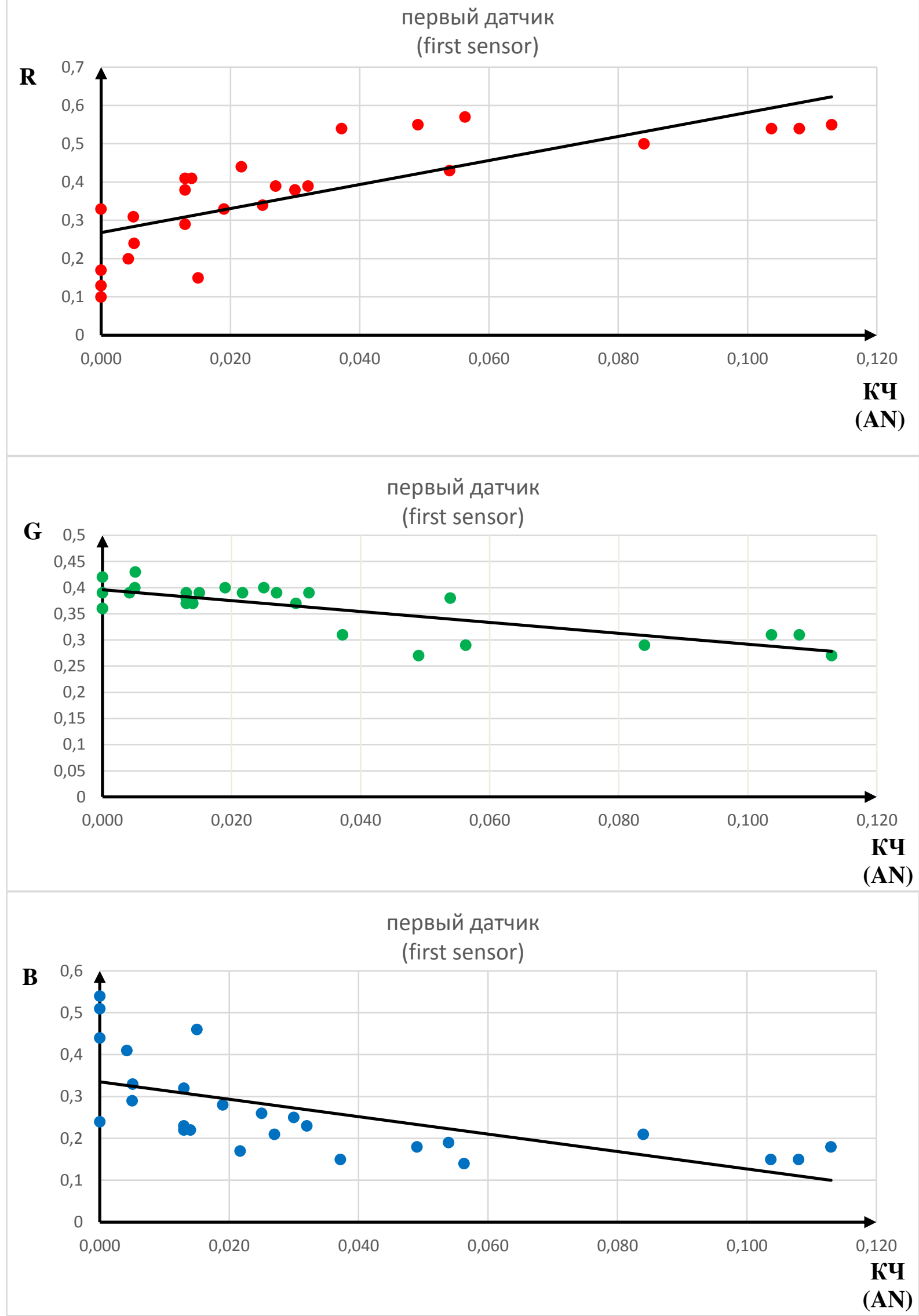

1) 

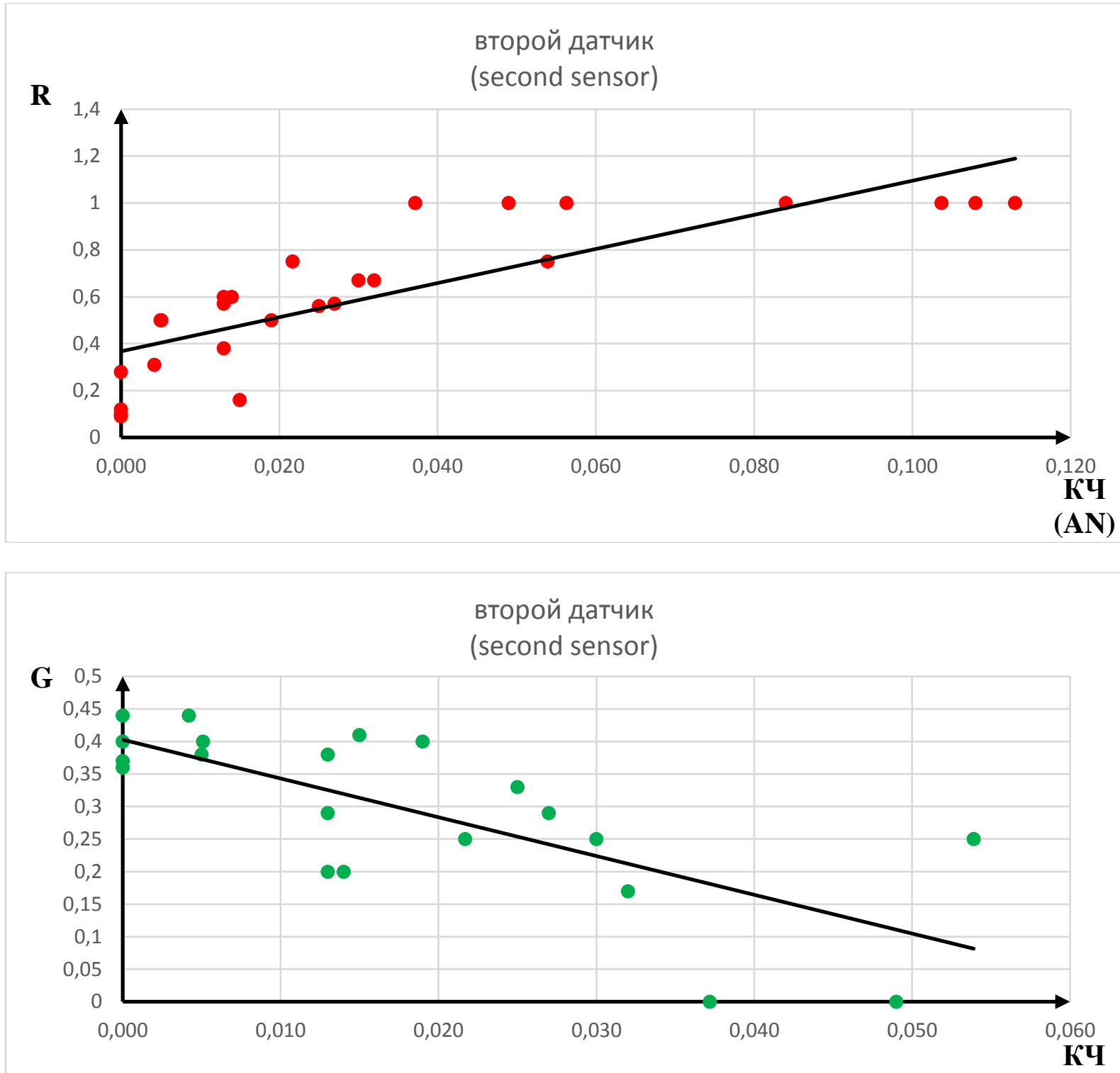

(AN)

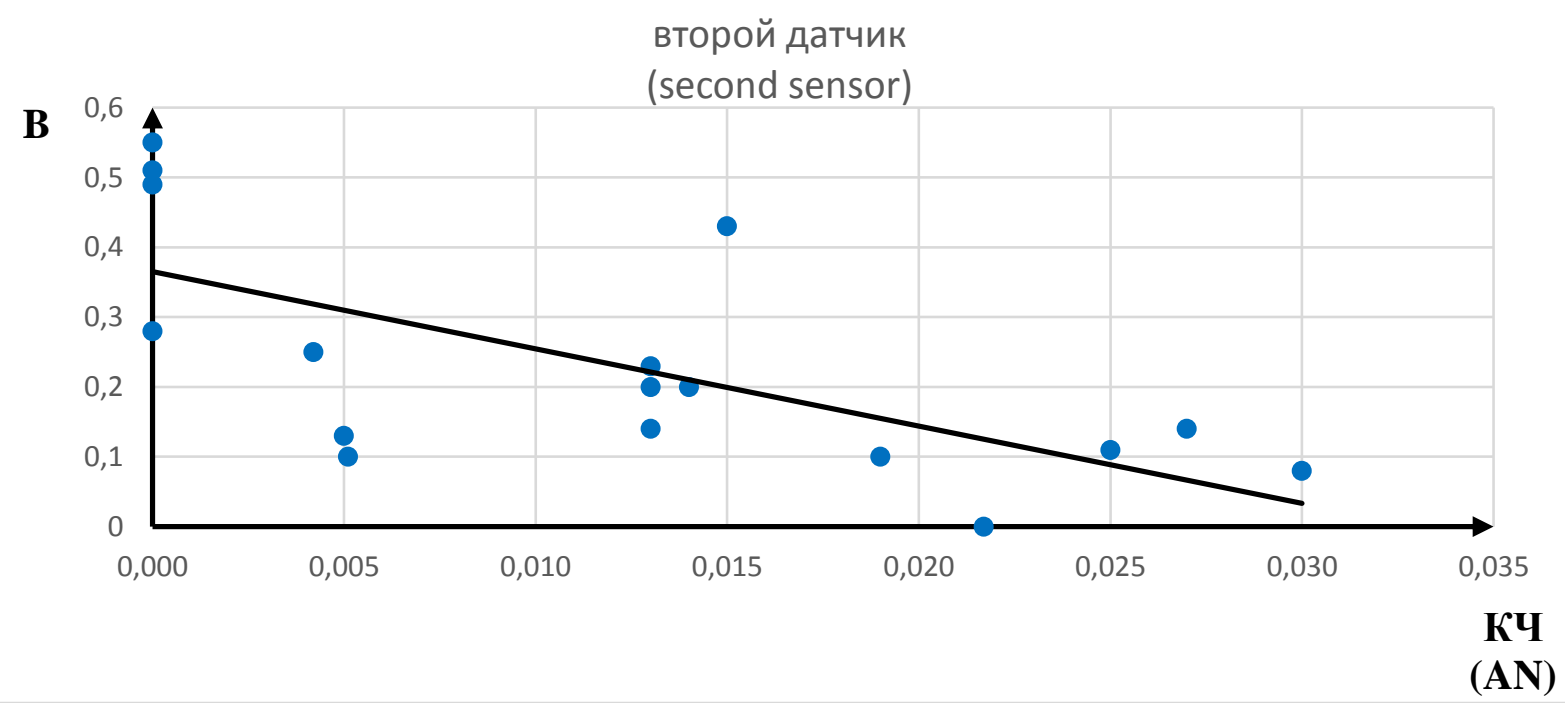

2) 


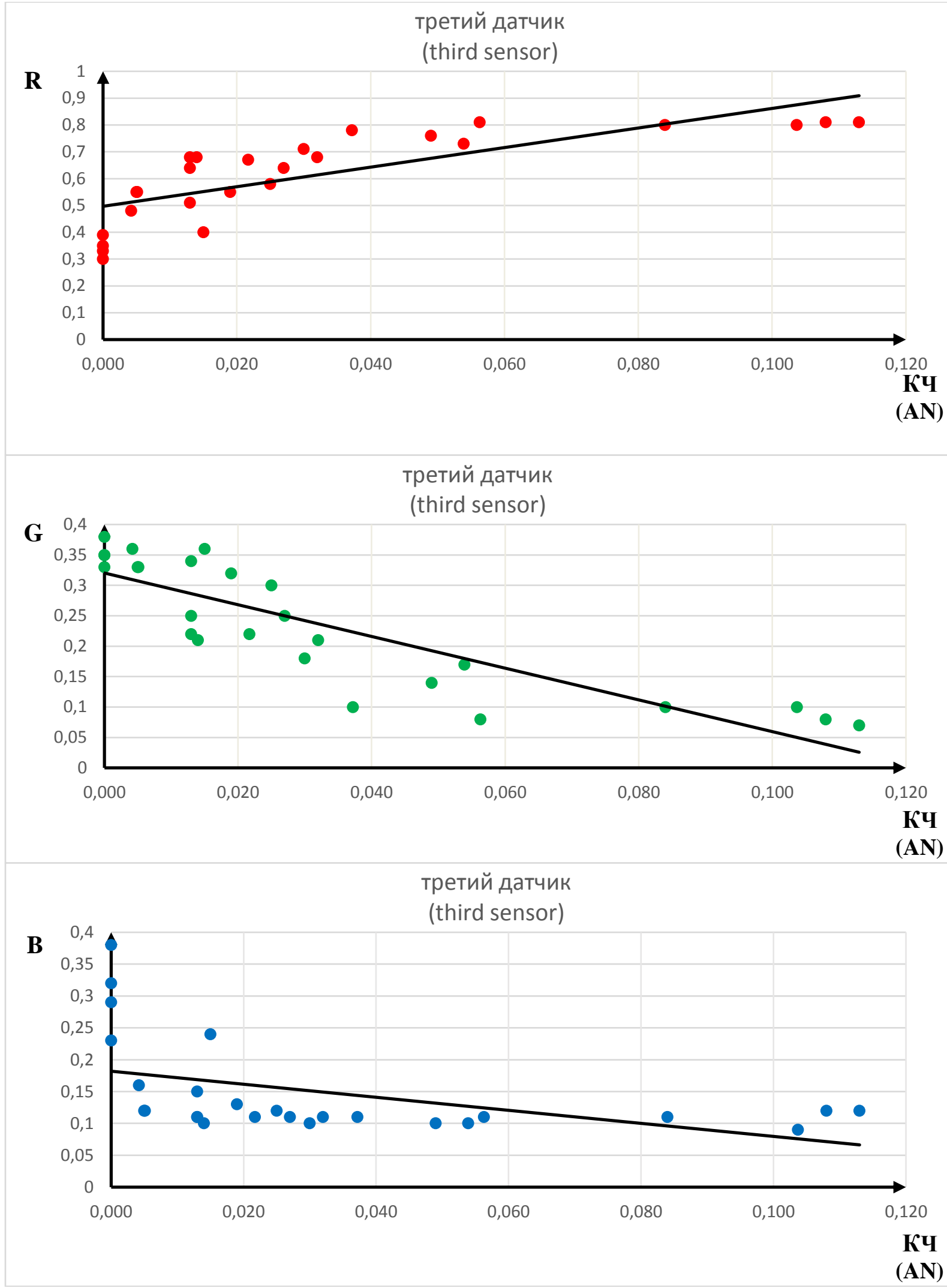

3)

Рис. 2. Зависимость координат цветности TM R, G, В от кислотного числа TM: 1 - первый датчик ; 2 - второй датчик; 3 - третий датчик

Fig. 2. Dependence of TM chromaticity coordinates $R, G, B$ on the $T M$ acid number: 1-first sensor; 2-second sensor; and 3-third sensor 
На рис. 3 приведены зависимости диэлектрических потерь ТМ, имеющие координат цветности $\mathrm{R}, \mathrm{G}, \mathrm{B}$ от тангенса угла достаточно сильную линейную корреляцию.
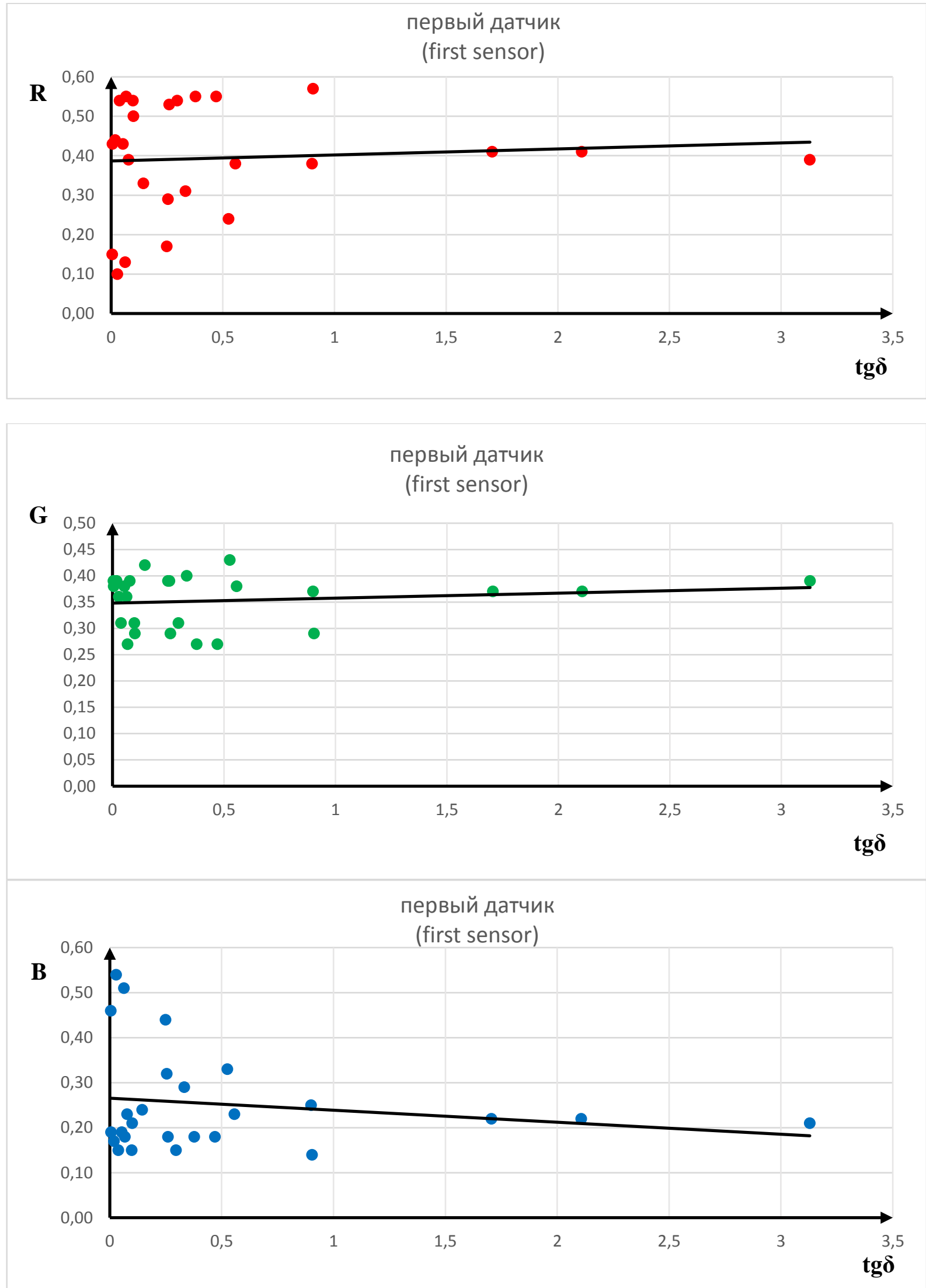

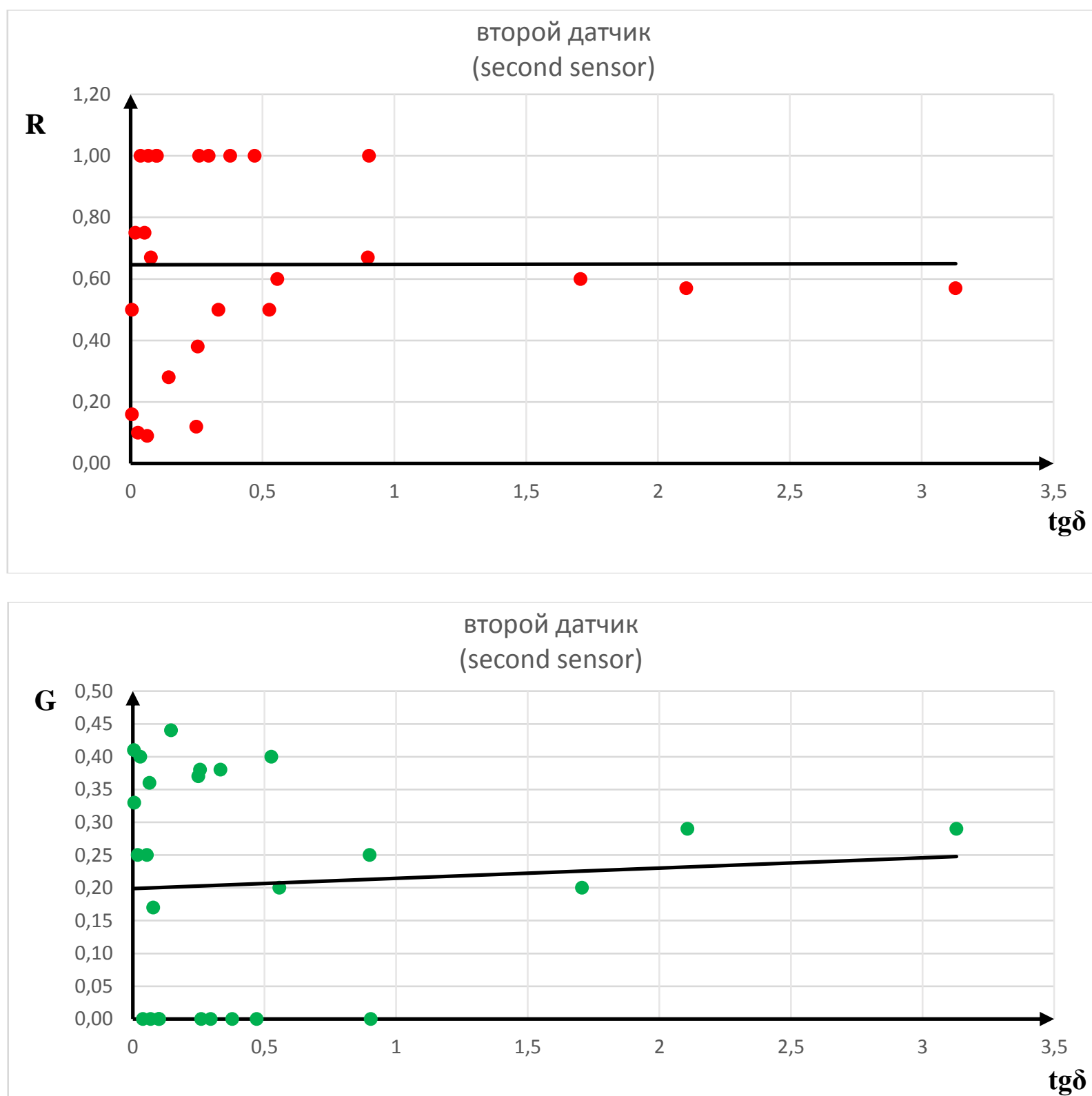

второй датчик

(second sensor)

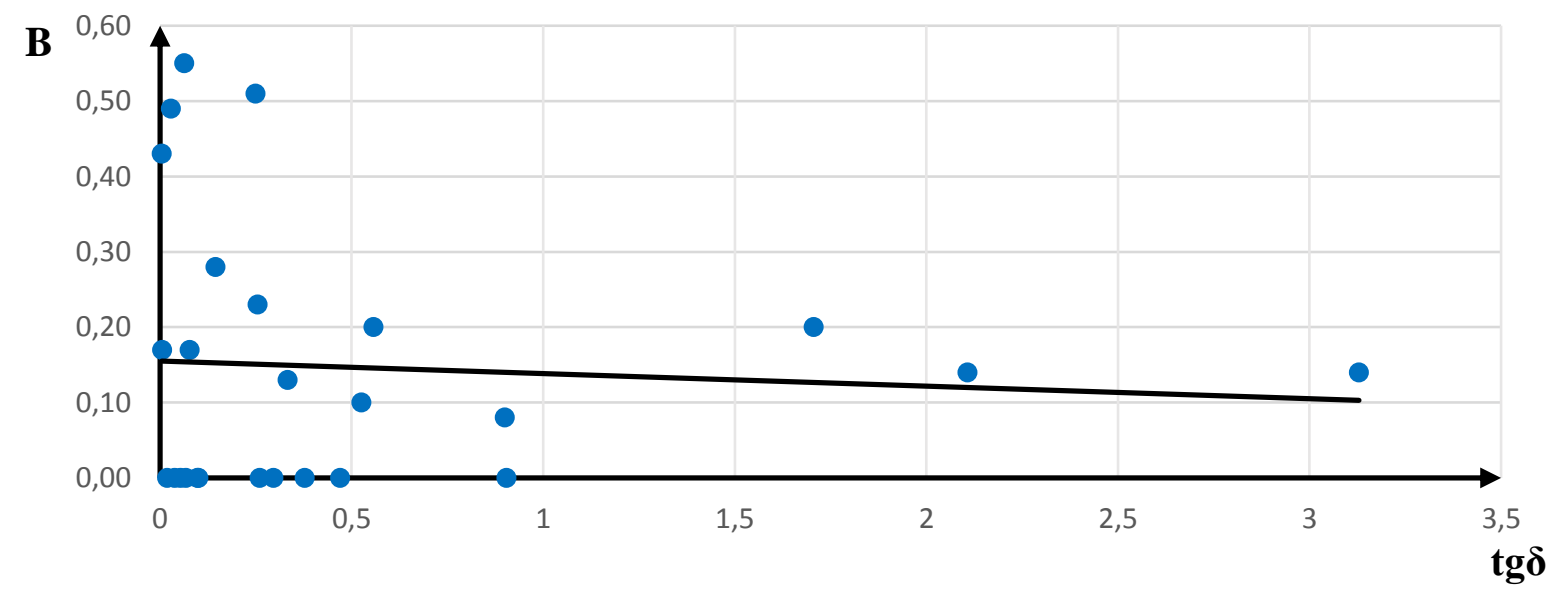



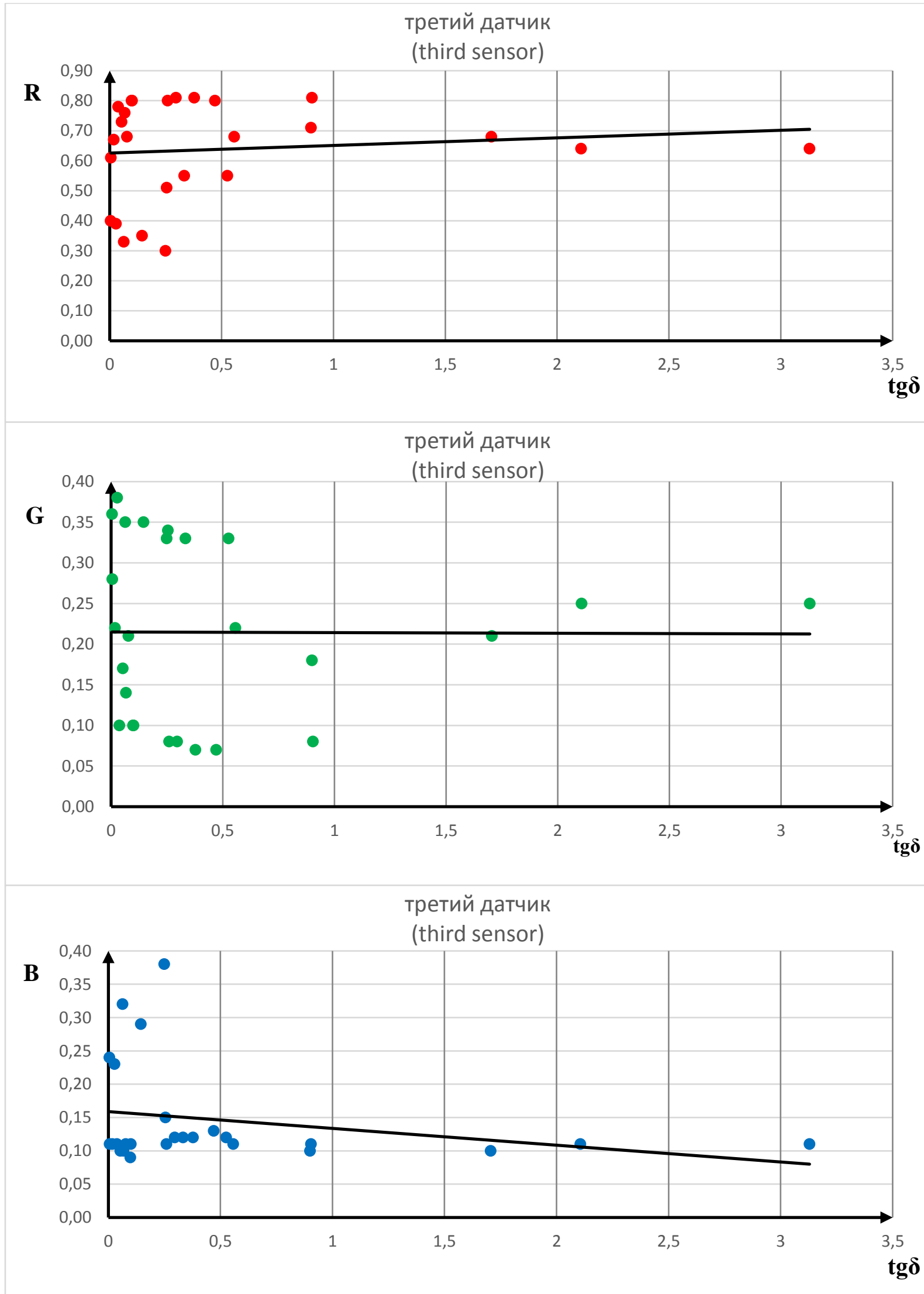

3)

Рис. 3. Зависимость координат цветности TM R, G, В от тангенса угла диэлектрических потерь ТМ: 1 - первый датчик ; 2 - второй датчик; 3 - третий датчик

Fig. 3. Dependence of TM chromaticity coordinates R, G, B on the tangent of the dielectric loss angle TM: 1-first sensor; 2 -second sensor; and 3-third sensor 
Как следует из рис. 3 лучшая корреляция для $\operatorname{tg} \delta$ наблюдается в области 1 датчика, а для кислотного числа, согласно рис. 2, в области датчика 2 и датчика 3 . По результатам таблицы 2 в программе «Statistica» построены линейные уравнения связывающие КЧ масла и $\operatorname{tg} \delta$ с соответствующими коэффициентами.

$$
K Y=0,23-0,165 \times R 3-0.39 \times G 3-0,09 \times B 3
$$

с коэффициентом корреляции равным 0,92 и $\operatorname{tg} \delta=20,19+29,36 \times R 1+12,84 \times G 1+24,95 \times B 1$ (2) с коэффициентом корреляции равным 0,95 .

Полученные уравнения (1) и (2) позволяют находить КЧ и $\operatorname{tg} \delta$ диэлектрических потерь ТМ по измеренным координатам цветности ТМ, т.е. по цвету масла.

\section{ВЫводы}

В результате проведенных исследований были получены следующие результаты:

- Исследованы цветовые характеристики ТМ и их зависимость от параметров каче-

\section{Литература (References)}

[1] Lipshtein R.A., Shakhnovich M.I. Transformatornoe maslo [Transformer oil]. Moscow, 1983, 296 p. (In Russian).

[2] Vysogorets S.P. Razrabotka metodiki expressanaliza parametra kachestva izolyacionnogo masla [Development of a methodology for express analysis of the quality parameter of insulating oil] Nadezhnost' i bezopasnost' energetiki - Dependability and safety of power engineering, 2018, no. 1. pp. 41-47. (In Russian).

[3] STO 70238424.27.100053-2013. Energeticheskiye masla $i$ maslokhozyaystva elektricheskikh stantsiy $i$ setey. Normy $i$ trebovaniya. [Power oils and oil facilities of power plants and networks. Norms and requirements.] Moscow: NP INVEL, 2nd edition, 2013, 159 p. (In Russian).

[4] $R D$ 34.45-51.300-97 Ob'em i normy ispytaniya energooborudovaniya [The scope and standards of testing electrical equipment], Moscow, 2001, 177 p. (In Russian).

[5] Poiss G., Vitolina S., Marks J. Development of a risk matrix considering specific features of the power transformer park of Latvia. Advances in Science, Technology and Engineering Systems Journal. 2018, vol. 3, no. 1, pp.148-154.

[6] Ohodnicki P.R., Stewart E.M. Chemical Sensing Strategies for Real-Time Monitoring of Transformer Oil. IEEE Sensors Journal, 2017. vol. 17, Iss. 8, pp. 5786-5806. doi: ства ТМ;

- Установлены корреляционные связи между координатами цветности ТМ их КЧ и $\operatorname{tg} \delta \mathrm{TM}$

- Получены корреляционные уравнения между координатами цветности ТМ их КЧ и $\operatorname{tg} \delta$ ТМ, позволяющие определять эти характеристики у исследуемых масел по их цветовым характеристикам.

Полученные результаты свидетельствуют о том, что по координатам цветности, можно определить КЧ и $\operatorname{tg} \delta$ ТМ. Это значительно облегчает диагностику трансформаторного масла, а также снижает стоимость исследования, так как для него не нужны никакие дополнительные приборы, кроме белого широко распространенного в быту светодиода и емкости для масла, что является важным фактором для электроэнергетики. Метод дает ценную информацию относительно его текущего состояния, что позволяет предотвратить его полное окисление и как следствие возможные причины аварий маслонаполненного оборудования.

\subsection{9/JSEN.2017.2735193}

[7] Vagapov T.R., Volkova T.A. [Transformer oil as a diagnostic medium for power transformers] Electrotechnical complexes and systems: Proceedings of the international. scientificpractical conf. Ufa, 2016, pp. 39-42. (In Russian).

[8] Lilan Liu, Hao Wu, Teqing Liu, Hua Feng, Huidong Tian, Zongren Peng. Influence of Moisture and Temperature on The Frequency Domain Spectroscopy Characteristics of Transformer Oil, Proc. of the IEEE International Conference on Dielectrics, 2016, vol. 1, pp. 565-568. doi:10.1109/ICD.2016.7547667

[9] Mitchinson P. M., Hosier I. L., Lewin P. L., Vaughan A. S., Chen G. C., Jarman P. An experiment to evaluate the benefits of processing aged transformer oil, Conference Record of the 2006 IEEE International Symposium on Electrical Insulation, 2006, pp. 89-92, doi: 10.1109/ELINSL.2006.1665264.

[10]Lelekakis N., Wijaya J., Martin D., Susa D. The effect of acid accumulation in power-transformer oil on the aging rate of paper insulation. IEEE Electrical Insulation Magazine, 2014, vol. 30, no. 3, pp. 19-26. doi: 10.1109/MEI.2014.6804738

[11] Karthik R., Sree Renga Raja T. Investigations of transformer oil characteristics. IEEJ Trans Elec Electron Eng, 2012 vol.7, pp. 369-374. doi: 10.1002/tee.21742

[12] Peng D., Yang D., Wang C., Li M., Research of the Dielectric Loss factor tan delta of Transform- 
er Oil by Multi-Parameter Regression Analysis. Asia-Pacific Power and Energy Engineering Conference, 2009, pp. 1-3, doi: 10.1109/APPEEC.2009.4918669.

[13] Singh, J. , Sood, Y. and Verma, P. Impact of Accelerated Stresses on Power Transformer Insulation. Energy and Power Engineering. 2017, vol. 9, Iss. 4, pp. 217-231. doi: 10.4236/epe.2017.94015.

[14] Mohamed A.A. Wahab, Hamada , M.M., Zeitoun A.G., Ismail G. Novel modeling for the prediction of aged transformer oil characteristics. Electric Power Systems Research, 1999, vol. 51, Iss. 1, pp. 61-70. doi: 10.1016/S03787796(98)00156-4

[15] Fofana I., Hadjadj Y. Electrical-Based Diagnostic Techniques for Assessing Insulation Condition in Aged Transformers. Energies, 2016, vol. 9, p. 679. doi:10.3390/en9090679.

[16] Kohtoh M., Kaneko S., Okabe S., Amimoto T., Aging effect on electrical characteristics of insulating oil in field transformer. IEEE Transactions on Dielectrics and Electrical Insulation, 2009, vol. 16, no. 6, pp. 1698-1706, doi: 10.1109/TDEI.2009.5361592.

[17] Forouhari S., Abu-Siada A. Remnant life estimation of power transformer based on IFT and acidity number of transformer oil. 2015 IEEE 11th International Conference on the Properties and Applications of Dielectric Materials (ICPADM), 2015, pp. 552-555. doi:10.1109/ICPADM.2015.7295331.

[18] Chen S., Zhu Y., Jia Y., Yun Y., Han Z., Feng G., Zhang L., Pang X. Study on analysis of acid number of transformer oil by temperature titration. IOP Conference Series: Earth and Environmental Science, 2019, vol. 300, Iss. 4, pp 2430.

[19] Peng D., Yang D., Wang C., Li M. Research of the Dielectric Loss factor tan delta of Transform-

\section{Сведения об авторах.}
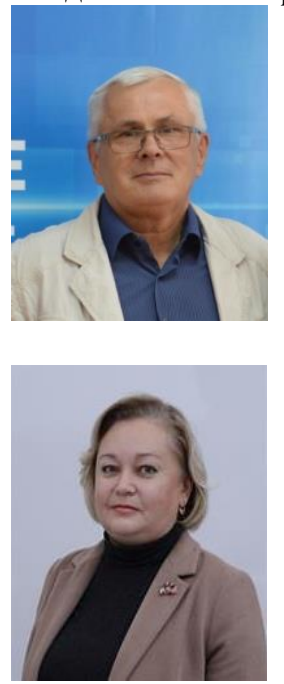

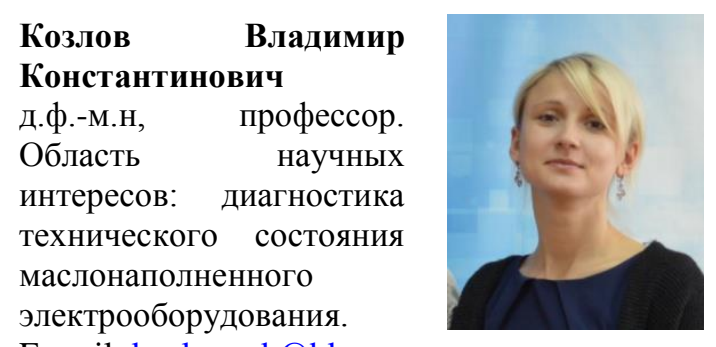

E-mail: kozlov vk@bk.ru

Валиуллина Дилия

Мансуровна, к.т.н, доцент. Область научных интересов: диагностика технического состояния маслонаполненного электрооборудования. E-mail: valiullinadiliya@mail.ru

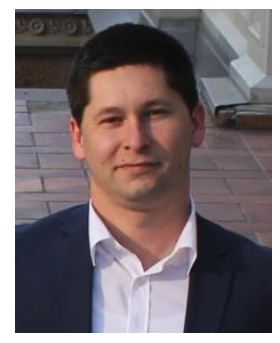

er Oil by Multi-Parameter Regression Analysis. 2009 Asia-Pacific Power and Energy Engineering Conference, 2009, pp. 1-3, doi:

10.1109/APPEEC.2009.4918669.

[20] Okabe S., Kaneko S., Kohtoh M., Amimoto T. Analysis results for insulating oil components in field transformers. IEEE Transactions on Dielectrics and Electrical Insulation, 2010, vol. 17, no. 1, pp. 302-311. doi: 10.1109/TDEI.2010.5412031.4.

[21] Tomin V.P., Korchevin E.N. Issledovaniye stareniya transformatornykh masel $\mathrm{v}$ atmosfere razlichnykh gazov pod vozdeystviyem elektricheskogo polya [Investigation of aging of transformer oils in an atmosphere of various gases under the influence of an electric field] Energetik. 2017, no. 5. pp. 25-28. (In Russian).

[22] Abdrashitov R.R. [Diagnostics and monitoring of power transformers]. Trudy mezhdunarodnoy naucho-prakticheskoy konferentsii ' Rol' $i$ mesto informatsionnykh tekhnologiy $v$ sovremennoy nauke" [Proc. international scientific and practical conference "'The role and place of information technologies in modern science"] Ufa, 2018, pp. 19-21. (In Russian).

[23] Syrtsov A.I., Polovinka D.V., Parsentiev O.S. Povysheniye nadezhnosti silovykh transformatorov putem sovershenstvovaniya ikh ispytaniy [Increasing the reliability of power transformers by improving their testing]. Prospects for the development of information technologies, 2016, no. 32, pp. 76-86. (In Russian).

[24] Shutenko O.V. Kompleksnyy korrelyatsionnyy analiz pokazateley kachestva transformatornogo masla [Comprehensive correlation analysis quality indicators transformer oil]. Vestnyk Nats. tekhn. un-ta "KHPY" - Newspaper Nat. tech. HPI University, Kharkov, 2008, vol. 45, pp.161-172. 